\title{
Editorials
}

\section{Enhancing ventilatory strategies for the critically ill - proportional assist ventilation}

Martin R. Lessard MD FRCPC

$\mathrm{P}$ ROPORTIONAL assist ventilation (PAV) is a new mode of synchronized partial ventilatory support designed to optimize adaptation of the ventilator to the patient. ${ }^{1}$ Proportional assist ventilation differs markedly from other modes of partial assistance. Rather than providing a fixed volume or pressure assistance to the patient, PAV generates pressure assistance in proportion to the patient's own respiratory effort. This unique characteristic has the potential to facilitate optimal adaptation of the ventilator to the patient's changing respiratory demand.

An appreciation of the physiological basis of assisted ventilation helps our understanding of the working principles of PAV. If one considers a patient's respiratory system impedance with its resistive and elastic components, pressure or effort must be generated by the patient (Pmus) and/or the ventilator (Pvent) for any given inspiration. The amount of effort required is dependent upon the elastance of the respiratory system $(\mathrm{E}=1 /$ compliance $)$ and the cumulative inspired volume, and on the total resistance of the patient's respiratory system and breathing apparatus (R) and the inspiratory flow rate. Partition of the respiratory effort between the patient and the ventilator varies according to the mode of ventilation. In the spontaneously breathing patient (e.g., continuous positive airway pressure mode), inspiratory Pvent is negligible, and all the inspiratory work is generated by the patient's respiratory muscles.

\section{(1) Ptotal $=$ Pmus $=($ Flow $x \mathrm{R})+($ Volume $\mathrm{x} E)$}

On the other hand, during controlled ventilation (e.g., anesthetized and paralyzed patient), the patient's respiratory muscles rest, and the ventilator provides the entire work of respiration.

\section{(2) Ptotal $=$ Pvent $=($ Flow $x$ R $)+($ Volume $x \mathrm{E})$}

However, most of the time in mechanically ventilated intensive care unit patients, the respiratory effort is partitioned between the patient and the ventilator in a complex and dynamic manner described by the following relationship:

(3) Ptotal $=$ Pvent + Pmus $=($ Flow $x$ R $)+($ Volume $\mathrm{x}$ E) (equation of motion)

For a typical patient with a known $\mathrm{E}$ and $\mathrm{R}$, during standard volume assist-control (VAC) mode (or mandatory breaths in synchronized intermittent ventilation mode), inspiratory flow and volume are fixed ventilator settings determined by the physician. Thus, ventilator assistance (Pvent) is a dependent variable that is reduced when the patient's spontaneous work of breathing increases, in an antagonistic relationship (see the equation of motion above). In pressure controlled modes (e.g., pressure controlled ventilation, pressure support), Pvent is a fixed setting, while flow and volume are dependent variables. Inspired volume and flow may vary with changing patient respiratory effort while ventilator assistance remains independent of the patient's inspiratory effort, therefore providing constant inspiratory support. ${ }^{2}$

Proportional assist ventilation differs from conventional pressure modes in that Pvent is not a pre-determined setting. Rather, the pressure assistance with PAV is dynamic, varying in linear proportion to the patient's spontaneous inspiratory effort. With pressure support ventilation (PSV), the level of support (pressure) is determined by the physician or respiratory therapist, while with PAV it is the proportion of the total respiratory effort to be provided by the ventila-

CAN J ANESTH $2007 / 54: 6 /$ pp 407-413

From the Department of Anesthesia and Critical Care, Centre hospitalier affilié universitaire de Québec, Université Laval, Québec, Québec, Canada.

Address correspondence to: Dr. Martin Lessard, Département d'anesthésie-réanimation, CHA (Hôpital de l'Enfant-Jésus), 1401, 18e rue, Québec, Québec GlJ 1Z4, Canada. Phone: 418-649-5807; Fax: 418-649-5918; E-mail: martin.lessard@anr.ulaval.ca The author has no conflict of interest pertaining to the subject of this manuscript. 
tor which is set, with no direct control over the pressure assistance. ${ }^{3}$ The rationale behind PAV is that the patient's respiratory center knows best what the respiratory requirements are, thus respiratory assistance should be limited to assisting the patient with minimal interference on the breathing pattern. Patient demand for more rapid or deeper breathing requires greater assistance, which he will receive. Conversely, patient demand for smaller breaths will result in a lower level of assistance, better matched to individual needs. As a result, PAV ensures a positive relationship between the degree of ventilator assistance and the patient's spontaneous effort, compared to volume controlled modes (inverse relationship) or pressure controlled modes (no relationship) (Figure).

From a physiological perspective, PAV incorporates many of the characteristics for the preferred mode of assistance for patients requiring partial ventilatory support. However, more than 15 years after its initial description, use of PAV has not been widely adopted in the clinical setting. ${ }^{4}$ Although its operational concept is appealing, PAV is also much more complex than either VAC or PSV. Proportional assist ventilation generates flow assistance and volume assistance in proportion to the patient's respiratory muscles effort against resistance and elastance respectively. Thus, the PAV ventilator software requires measurements of the instantaneous inspiratory flow and volume, as well as respiratory system resistance and elastance. Instantaneous flow and volume measurements are easily obtained by rapid response flow sensors on modern ventilators. On the other hand, total resistance and elastance are usually obtained during constant flow volume controlled ventilation in a relaxed or sedated patient. But this setting differs markedly from that of the spontaneously breathing patient, and can yield inaccurate measurements. An alternative method (the runaway method) to measure resistance and elastance in spontaneously breathing patients has been proposed, but it requires additional expertise and some training. ${ }^{5}$ Errors in the measurement of resistance and elastance are not trivial, and can lead to under-assistance or over-assistance (runaway phenomenon). ${ }^{1,5}$ Finally, things get even more problematic with an intubated patient, since resistance of the endotracheal or tracheotomy tube adds to resistive load of the patient's respiratory system. The equation of motion of an intubated patient then rewrites as follows:

(4) Ptotal $=$ Pvent + Pmus $=($ Flow $x$ Ret $)+($ Flow $\mathrm{x}$ Rrs $)+($ Volume $\mathrm{x}$ E)

where Ret is the resistance of the tracheal tube, which varies according to its length, diameter and to the

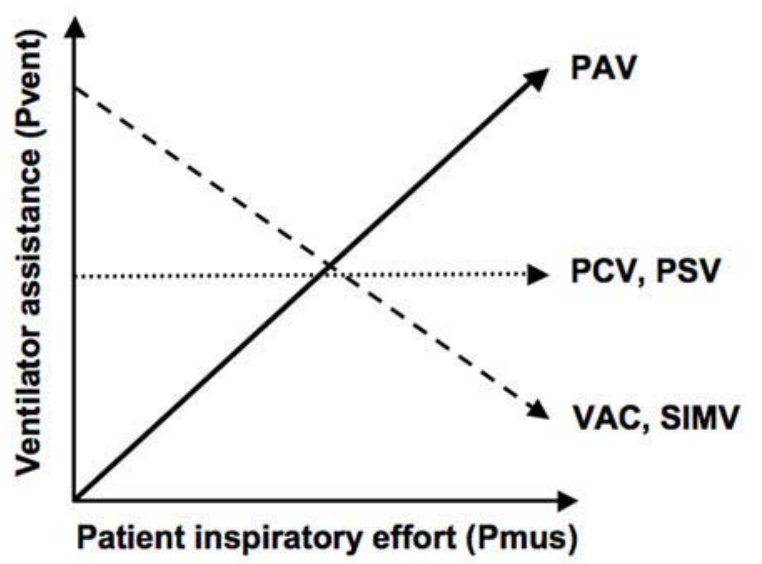

FIGURE The relationship between patient spontaneous inspiratory effort and ventilator assistance is markedly different with different modes of ventilation. In volume controlled modes, assistance provided by the ventilator decreases with increasing patient effort, while in pressure controlled modes, ventilator assistance is unaffected by patient effort. In proportional assist ventilation, ventilator assistance is a positive function of the patient inspiratory effort. VAC = volume assist-control, SIMV = synchronized intermittent mandatory ventilation, $\mathrm{PCV}=$ pressure controlled ventilation, PSV = pressure support ventilation, PAV $=$ proportional assist ventilation.

inspiratory flow, while Rrs is the resistance of the patient's respiratory system (lung and thorax) which is mostly independent of flow. Because resistance of tracheal tubes (Ret) varies significantly with flow, PAV (whose working principle requires a constant resistance) will poorly compensate for Ret. ${ }^{5-7}$ One way to accommodate the tracheal tube resistive load is to use modes such as the automated tube compensation, which computes the ventilator pressure required to compensate for the resistance of the tracheal tube for every breath. However, there are limitations with these systems which do not always perform as expected, yielding suboptimal compensation of the endotracheal tube resistance. ${ }^{8}$

In a study reported in this issue of the Journal, Mirkovic et al. address some of these issues with the implementation of PAV in the clinical setting, bringing two modifications to the conventional PAV system. ${ }^{9}$ First, they used a simplified version of PAV, volume assist ventilation (VAV), providing assistance solely for the elastic component of the respiratory system impedance without any assistance for the resis- 
tive component. Secondly, they moved the site of airway pressure measurement from the Y-piece of the breathing circuit to the distal end of the tracheal tube. With this last modification, the PAV ventilator is triggered and regulated by tracheal pressure fluctuation rather than by pressure fluctuation in the breathing circuit. $^{10}$

The methodology used by Mirkovic et al. to measure the effects of their system on the patients' work of breathing may appear somewhat complex to those less familiar with such measurements. Assessment of patient's inspiratory effort and ventilator assistance were calculated by the pressure-time integral, which is analogous to the calculation of work of breathing from Campbell diagrams. ${ }^{11,12}$ This is standard methodology for assessing a patient's inspiratory effort during mechanical ventilation, allowing for partitioning of the total inspiratory effort into its chest wall related elastic, lung related elastic and resistive components, as well as effort related to dynamic hyperinflation (intrinsic positive end-expiratory pressure). This is illustrated in Figure I of Mirkovic's paper: panel A showing the inspiratory effort (partitioned into its components) of a typical patient with airway opening pressure regulation (left) and with tracheal pressure regulation (right), panel $\mathrm{B}$ showing the ventilator assistance provided by VAV with the same two regulation systems. ${ }^{9}$

Unsurprisingly, VAV progressively reduced the patients' elastic effort with increasing level of assistance. More interestingly, tracheal pressure regulated ventilation provided an important assistance against resistive loads compared with the conventional system (airway opening pressure regulation). It can be clearly seen in Mirkovic's Figure 1 that, with tracheal pressure regulation, a large part of the resistive effort was "transferred" from the patient to the ventilator. This increase in resistive assistance provided by the ventilator is also shown in Figure 4. Tracheal pressure regulation translated into an approximately $50 \%$ reduction in the patients' resistive effort at all levels of VAV (shown in Figure 3).

Mirkovic et al. are to be commended for proposing a simplified and easier to use PAV system. However, their approach has several limitations. Resistance of the endotracheal tube (which was effectively abolished with tracheal pressure regulation) comprised only about $50 \%$ of the total resistive component of inspiratory work. What about the remainder of the resistive load? Even at high levels of volume assistance (VAV 80\%), the remaining resistive effort was still significant, equivalent to the elastic effort without any volume assistance (VAV 0\%). The study population was small (seven patients) of whom only two had a history of obstructive lung disease with elevated respiratory system resistance. Others have reported that flow assistance against resistive loads is an important component to optimize PAV. ${ }^{13}$ Obviously, more data are required in patients with obstructive lung diseases. Furthermore, Mirkovic et al. tested their system in a controlled research setting for only a two-hour duration. Tracheal pressure triggering and regulation requires the use of specially designed endotracheal tubes. Maintaining patency of the tracheal pressure measurement lumen, and thus ensuring the functionality of this modified PAV system over days could be problematic in the clinical setting.

Proportional assist ventilation, whether in its original or simplified version, has many questions left unanswered. Compared to other modes of partial ventilatory support, a clear outcome benefit to the patient (apart from improved comfort) has yet to be demonstrated. Additionally, as mentioned above, measurement of patient respiratory mechanics is not easily accomplished in the clinical setting. Continuous automated measurement of respiratory system resistance and elastance (or compliance) has been recently described and validated. ${ }^{14,15}$ The new PAV+ ${ }^{\circledR}$ software available on the Puritan-Bennett 840 ventilator takes advantage of such automated measurements. ${ }^{16}$ Still, the optimal level of assistance of the patient's effort is unknown. Easy to use criteria for assessing patient tolerance, and for titration and weaning of PAV are also lacking.

In conclusion, although the tracheal pressure regulated volume assist ventilation system described by Mirkovic et al. requires further evaluation in a larger series of patients with various pathologies, their novel work is clearly aiming in the right direction: to render user-friendly, a complex mode of partial ventilatory support. Original approaches such as theirs provide innovative paths to resolving the challenges of implementing PAV in the clinical setting. 


\section{L'amélioration des stra-} tégies respiratoires pour les patients sévèrement malades - la ventilation assistée proportionnelle

La ventilation assistée proportionnelle (proportional assist ventilation - PAV) est un nouveau mode d'assistance respiratoire partielle synchronisée conçue pour optimiser l'adaptation du respirateur au patient. ${ }^{1}$ La ventilation assistée proportionnelle diffère sensiblement des autres modes d'assistance partielle. En effet, au lieu de fournir au patient une pression d'aide ou un volume fixes, la PAV génère une pression d'aide proportionnelle à l'effort respiratoire du patient. Cette caractéristique unique peut potentiellement faciliter une adaptation optimale du respirateur à la demande respiratoire fluctuante du patient.

Une connaissance des bases physiologiques de la ventilation assistée nous permet de mieux comprendre les principes de fonctionnement de la PAV. Compte tenu de l'impédance du système respiratoire d'un patient avec ses composantes résistives et élastiques, une pression ou effort doit être généré par le patient (Pmus) et/ou le ventilateur (Pvent) à chaque inspiration. La quantité d'effort requis dépend de l'élastance du système respiratoire ( $\mathrm{E}=1$ /compliance) et du volume inspiré cumulatif, ainsi que de la résistance totale du système respiratoire du patient et de l'appareillage respiratoire (R) et du débit inspiratoire. La répartition de l'effort respiratoire entre le patient et le ventilateur varie selon le mode de ventilation. Chez le patient respirant spontanément (par ex., mode de pression positive continue, CPAP), la Pvent inspiratoire est négligeable, et tout le travail de l'inspiration est généré par les muscles respiratoires du patient.

$$
\text { (1) Ptotal }=\text { Pmus }=(\text { Débit } \mathrm{x} R)+(\text { Volume } \mathrm{x} E)
$$

En revanche, lors de la ventilation contrôlée (par ex., chez un patient anesthésié et paralysé), les muscles respiratoires du patient sont au repos, et le ventilateur fournit tout le travail de la respiration.

(2) Ptotal $=$ Pvent $=($ Débit $x \mathrm{R})+($ Volume $\mathrm{x} E)$

Toutefois, chez des patients des soins intensifs ventilés mécaniquement, l'effort respiratoire est la plupart du temps réparti entre le patient et le ventilateur selon un mode complexe et dynamique, décrit par la relation suivante :

(3) Ptotal $=$ Pvent + Pmus $=($ Débit $x \mathrm{R})+($ Volume $\mathrm{x} \mathrm{E})$ (équation du mouvement de l'air)

Chez un patient typique dont $\mathrm{E}$ et $\mathrm{R}$ sont connus, avec le mode de ventilation assistée contrôlée volumétrique (VAC) (ou lors des cycles imposés de la ventilation intermittente, VACI), le débit et le volume inspiratoires sont des réglages fixes du ventilateur, déterminés par le médecin. Ainsi, l'assistance inspiratoire du ventilateur (Pvent) est une variable dépendante qui diminue lorsque l'effort respiratoire spontané du patient augmente, créant une relation antagoniste (voir l'équation du mouvement de l'air ci-dessus). Dans les modes à pression contrôlée (par ex., la ventilation en pression contrôlée, l'aide inspiratoire), la Pvent est un réglage fixe, alors que le débit et le volume sont des variables dépendantes. Le volume et le débit inspirés peuvent varier avec l'effort respiratoire changeant du patient, alors que l'assistance du ventilateur demeure indépendante de l'effort inspiratoire du patient, fournissant ainsi une assistance inspiratoire constante. ${ }^{2}$

La ventilation assistée proportionnelle diffère des modes de pression traditionnels en ce que la Pvent n’y est pas un réglage prédéterminé. Dans le cas de la PAV, la pression d'aide est dynamique et varie en proportion linéaire de l'effort inspiratoire spontané du patient. Avec le mode d'aide inspiratoire (pressure support ventilation), le niveau d'assistance (pression) est déterminé par le médecin ou par l'inhalothérapeute, alors qu'avec la PAV on détermine plutôt la proportion de l'effort respiratoire total à être fourni par le ventilateur, et il n'y a aucun contrôle direct sur le niveau de pression d'aide. ${ }^{3}$ Le raisonnement derrière le principe de la PAV est que le système respiratoire du patient est le plus apte à reconnaitre les besoins respiratoires, et que le support respiratoire devrait donc se limiter à aider le patient en dérangeant le moins possible le schéma de la respiration. Une respiration plus rapide ou plus profonde nécessite une aide plus élevée, et le patient la recevra. De même, en présence d'une demande respiratoire plus faible, l'aide sera moins élevée et mieux adaptée aux besoins individuels. Ainsi, comparée aux modes de volume contrôlé (relation inverse) ou aux modes de pression contrôlée (pas de relation), la PAV assure une relation positive entre le degré d'aide du respirateur et l'effort spontané du patient (Figure).

D'un point de vue physiologique, la PAV incorpore de nombreuses caractéristiques d'un mode 
d'assistance préféré pour les patients nécessitant un soutien respiratoire partiel. Toutefois, plus de 15 ans après sa description, l'utilisation de la PAV n'a pas encore été bien adoptée dans le milieu clinique. ${ }^{4}$ Bien que son concept opérationnel soit séduisant, la PAV est également bien plus complexe que la VAC ou l'aide inspiratoire. La ventilation assistée proportionnelle génère une assistance au débit inspiratoire et une assistance au volume courant inspiré proportionnellement à l'effort respiratoire spontané du patient contre la résistance et l'élastance, respectivement. Donc, le logiciel du ventilateur pour la PAV nécessite la mesure du débit et du volume inspiratoires instantanés ainsi que de la résistance et de l'élastance du système respiratoire. Les mesures instantanées du débit et du volume sont facilement obtenues dans les ventilateurs modernes par des capteurs de débit à réponse rapide. En revanche, la résistance et l'élastance sont en général obtenues pendant la ventilation contrôlée à débit et volume constants sur un patient paralysé ou sous sédation. Mais cette situation diffère sensiblement de celle du patient respirant spontanément, ce qui peut engendrer des mesures inexactes. Une autre méthode (la méthode de runaway) pour la mesure de la résistance et de l'élastance chez des patients respirant spontanément a été décrite, mais elle requiert une très bonne expertise. ${ }^{5}$ Les erreurs dans la mesure de la résistance et de l'élastance ne sont pas sans conséquence et peuvent mener à une sous-assistance ou au contraire une sur-assistance (phénomène de runaway)., ${ }^{1,5}$ Finalement, cela devient encore plus problématique chez un patient intubé, étant donné que la résistance du tube endotrachéal ou de trachéotomie s'additionne à la résistance du système respiratoire du patient. L'équation du mouvement de l'air d'un patient intubé s'écrit alors de la manière suivante :

(4) Ptotal $=$ Pvent + Pmus $=($ Débit $x$ Ret $)+($ Débit $\mathrm{x}$ Rrs $)+($ Volume $\mathrm{x} E)$

où Ret correspond à la résistance du tube trachéal, qui varie selon sa longueur, son diamètre et le débit inspiratoire, et où Rrs correspond à la résistance du système respiratoire du patient (poumon et thorax), laquelle est en majeure partie indépendante du débit. Parce que la résistance des tubes trachéaux (Ret) varie considérablement selon le débit, la PAV (dont le principe de fonctionnement nécessite une résistance constante) ne compensera par correctement la Ret. ${ }^{5-7}$ Une façon d'éliminer la charge résistive du tube trachéal est d'utiliser des modes tels que la compensation de tube automatique (automatic tube compensation), qui calcule la pression inspiratoire requise afin de compenser

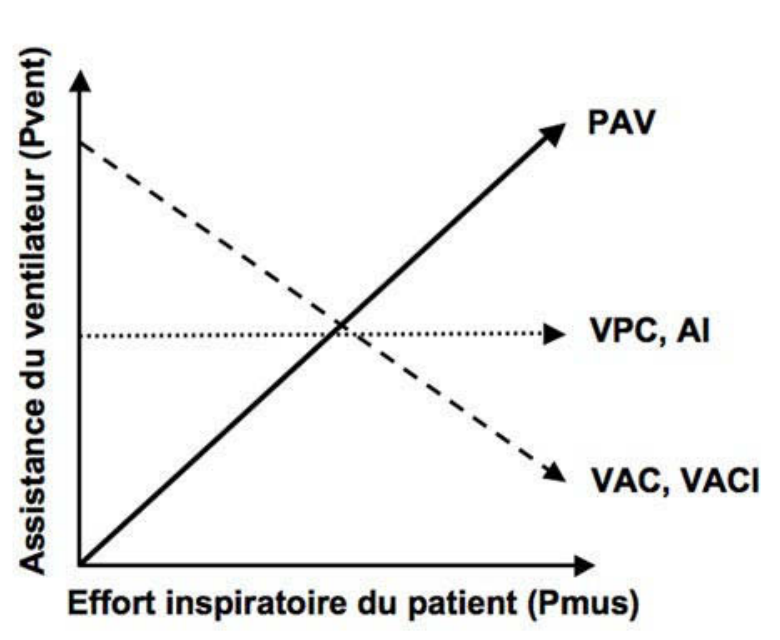

FIGURE La relation entre l'effort inspiratoire spontané d'un patient et l'assistance du ventilateur est très différente selon les différents modes de ventilation. Dans les modes de volume contrôlé, l'assistance fournie par le ventilateur diminue lorsque l'effort respiratoire spontané du patient augmente, tandis que dans les modes de pression contrôlée, l'assistance du ventilateur n'est pas influencée par l'effort du patient. Dans le mode ventilation assistée proportionnelle, l'assistance du ventilateur est une fonction directe de l'effort inspiratoire du patient. VAC = ventilation assistée contrôlée volumétrique; VACI $=$ ventilation assistée contrôlée intermittente; $\mathrm{VPC}=$ ventilation en pression contrôlée; $\mathrm{AI}=$ aide inspiratoire $; \mathrm{PAV}=$ ventilation assistée proportionnelle.

la résistance du tube trachéal à chaque respiration. Toutefois, ces systèmes ont des limites et ne fonctionnent pas toujours tel qu'attendu, fournissant alors une compensation sous-optimale à la résistance du tube endotrachéal. ${ }^{8}$

Dans une étude rapportée dans ce numéro du Journal, Mirkovic et coll. abordent certains des problèmes associés à la mise en œuvre de la PAV dans un contexte clinique, et apportent deux modifications au système de PAV conventionnel. ${ }^{9}$ En premier lieu, ils utilisent une version simplifiée de PAV, la ventilation assistée proportionnelle au volume (VAV), fournissant une aide uniquement à la composante élastique de l'impédance du système respiratoire, sans aucune assistance à la composante résistive. Deuxièmement, les auteurs ont déplacé le site de mesure de la pression aérienne de la pièce en $\mathrm{Y}$ du circuit respiratoire à l'extrémité distale du tube trachéal. Grâce à cette deuxième modification, le ventilateur PAV est déclenché et régulé par les fluctuations de la pression trachéale plutôt que par celles de la pression dans le circuit respiratoire. ${ }^{10}$ 
La méthodologie utilisée par Mirkovic et coll. afin de mesurer les effets de leur système sur le travail respiratoire des patients peut apparaitre quelque peu complexe à ceux qui sont peu familiers avec ces mesures. L'évaluation de l'effort respiratoire du patient et de l'assistance fournie par le ventilateur a été calculée par l'intégrale pression-temps, qui est analogue au calcul du travail respiratoire selon les diagrammes de Campbell. ${ }^{11,12}$ Il s'agit d'une méthodologie standard pour la mesure de l'effort respiratoire d'un patient pendant la ventilation mécanique, permettant de séparer l'effort respiratoire total entre ses composantes élastiques liées à la paroi thoracique, et ses composantes élastiques et résistives associées aux poumons, ainsi que l'effort lié à l'hyperinflation dynamique (pression expiratoire positive intrinsèque). Ceci est illustré dans la figure 1 de l'article de Mirkovic: le panneau A montre l'effort inspiratoire (réparti en ses composantes) d'un patient typique avec régulation de la ventilation par la pression du circuit respiratoire (gauche) et avec régulation par la pression trachéale (droite) ; le panneau B montre l'aide respiratoire fournie par la VAV avec les deux mêmes systèmes de régulation. ${ }^{9}$

Comme on pouvait s'y attendre, la VAV a réduit de façon progressive l'effort élastique du patient avec l'augmentation séquentielle du niveau d'assistance. Ce qui est intéressant, c'est que la ventilation régulée par la pression trachéale a fourni une aide importante contre les charges résistives par rapport au système conventionnel (régulation par la pression du circuit). Dans la figure 1 de Mirkovic, nous voyons clairement qu'avec la régulation par la pression trachéale, une partie importante de l'effort résistif a été «transféré » du patient au respirateur. Cette augmentation de l'aide résistive fournie par le respirateur est également montrée dans la figure 4. La régulation de la VAV par la pression trachéale s'est traduite par une réduction d'environ $50 \%$ de l'effort résistif du patient et ce, à tous les niveaux d'assistance en VAV (illustré à la figure 3 ).

Mirkovic et coll. peuvent être félicités de proposer un système plus simple et facile d'utilisation de la PAV. Toutefois, leur approche comporte plusieurs limites. La résistance du tube endotrachéal (supprimée efficacement grâce à la régulation par la pression trachéale) ne correspondait qu'à environ $50 \%$ de la composante résistive totale du travail respiratoire. Qu'en est-il du reste de la charge résistive ? Même à des niveaux élevés d'assistance proportionnelle au volume (VAV 80 \%), l'effort résistif restant était encore important et équivalait à l'effort élastique en l'absence d'assistance proportionnelle au volume (VAV 0 \%).
La population à l'étude était petite (sept patients), dont seulement deux sujets avaient des antécédents de maladie pulmonaire obstructive avec une résistance élevée du système respiratoire. D'autres auteurs ont montré que la ventilation assistée proportionnelle au débit est une composante importante pour optimiser la PAV. ${ }^{13}$ Il va sans dire que des données supplémentaires sont nécessaires en ce qui concerne les patients souffrant de maladies pulmonaires obstructives. De plus, Mirkovic et coll. ont testé leur système dans un environnement de recherche contrôlé pour une durée de deux heures seulement. Le déclenchement et la régulation par la pression trachéale requièrent l'utilisation de tubes endotrachéaux spécialement conçus. Le maintien durant plusieurs jours de la perméabilité de la lumière de mesure de la pression trachéale, assurant ainsi le fonctionnement de ce système modifié de PAV, pourrait s'avérer problématique dans un contexte clinique.

La ventilation assistée proportionnelle, dans sa version originale ou simplifiée, comporte de nombreuses questions qui sont encore sans réponse. Par rapport à d'autres modes d'assistance respiratoire partielle, un bénéfice clair pour le patient (hormis un meilleur confort) n'a pas encore été démontré. En outre, comme cela a été mentionné plus haut, les mesures des paramètres de mécanique respiratoire des patients ne sont pas aisées dans le contexte clinique habituel. La mesure automatisée et continue de la résistance et de l'élastance (ou compliance) du système respiratoire a été récemment décrite et validée. ${ }^{14,15}$ Le nouveau logiciel PAV $+{ }^{\circledR}$ disponible sur le ventilateur PuritanBennett 840 tire profit de ces mesures automatisées. ${ }^{16}$ Néanmoins, le niveau optimal d'assistance à l'effort respiratoire du patient demeure inconnu. Des critères simples à utiliser pour évaluer la tolérance du patient et pour la titration et le sevrage de la PAV font également défaut.

En conclusion, bien que le système de ventilation assistée proportionnelle au volume régulé par la pression trachéale décrit par Mirkovic et coll. nécessite une évaluation plus poussée sur un nombre plus grand de patients souffrant de diverses pathologies, leur travail novateur vise clairement dans la bonne direction : rendre plus facile d'emploi un moyen complexe d'assistance respiratoire partielle. Des approches originales telle que la leur fournissent de nouvelles avenues de solution pour la mise en œuvre de la PAV dans la pratique clinique.

\section{References}

1 Younes $M$. Proportional assist ventilation, a new approach to ventilatory support. Theory. Am Rev 
Respir Dis 1992; 145: 114-20.

2 Brochard L, Harf A, Lorino H, Lemaire F. Inspiratory pressure support prevents diaphragmatic fatigue during weaning from mechanical ventilation. Am Rev Respir Dis 1989; 139: 513-21.

3 Younes $M$, Puddy A, Roberts D, et al. Proportional assist ventilation. Results of an initial clinical trial. Am Rev Respir Dis 1992; 145: 121-9.

4 Esteban A, Anzueto A, Alia I, et al. How is mechanical ventilation employed in the intensive care unit? An international utilization review. Am J Respir Crit Care Med 2000; 161: 1450-8.

5 Younes $M$. Proportional assist ventilation. In: Tobin MJ (Ed.). Principles and Practice of Mechanical Ventilation, 1st ed. New York: McGraw-Hill Inc.; 1994: 349-69.

6 Guttmann J, Eberhard L, Fabry B, Bertschmann W, Wolff $G$. Continuous calculation of intratracheal pressure in tracheally intubated patients. Anesthesiology 1993; 79: 503-13.

7 Varelmann D, Wrigge H, Zinserling J, Muders T, Hering R, Putensen C. Proportional assist versus pressure support ventilation in patients with acute respiratory failure: cardiorespiratory responses to artificially increased ventilatory demand. Crit Care Med 2005; 33: 1968-75.

8 Elsasser S, Guttmann J, Stocker R, Mols G, Priebe HJ, Haberthur C. Accuracy of automatic tube compensation in new-generation mechanical ventilators. Crit Care Med 2003; 31: 2619-26.

9 Mirkovic T, Paver-Erzen V Klokocovnik T, Gursabaney A, Hernandez P, Gottfried SB. Tracheal pressure regulated volume assist ventilation in acute respiratory failure. Can J Anesth 2007; 54: 420-9.

10 Banner MJ, Blanch PB, Gabrielli A. Tracheal pressure control provides automatic and variable inspiratory pressure assist to decrease the imposed resistive work of breathing. Crit Care Med 2002; 30: 1106-11.

11 Sassoon CS, Light RW, Lodia R, Sieck GC, Mahutte $C K$. Pressure-time product during continuous positive airway pressure, pressure support ventilation, and Tpiece during weaning from mechanical ventilation. Am Rev Respir Dis 1991; 143: 469-75.

12 Tobin MJ. Monitoring respiratory mechanics in spontaneously breathing patients. In: Tobin MJ (Ed.). Principles and Practice of Intensive Care Monitoring, 1st ed. New York: McGraw-Hill Inc.; 1998: 617-54.

13 Ranieri VM, Grasso S, Mascia L, et al. Effects of proportional assist ventilation on inspiratory muscle effort in patients with chronic obstructive pulmonary disease and acute respiratory failure. Anesthesiology 1997; 86: 79-91.

14 Younes M, Kun J, Masiowski B, Webster K, Roberts D.
A method for noninvasive determination of inspiratory resistance during proportional assist ventilation. Am J Respir Crit Care Med 2001; 163: 829-39.

15 Younes M, Webster K, Kun J, Roberts D, Masiowski B. A method for measuring passive elastance during proportional assist ventilation. Am J Respir Crit Care Med 2001; 164: 50-60.

16 Kondili E, Prinianakis G, Alexopoulou C, Vakouti E, Klimathianaki M, Georgopoulos D. Respiratory load compensation during mechanical ventilation-proportional assist ventilation with load-adjustable gain factors versus pressure support. Intensive Care Med 2006; 32: 692-9. 